\title{
El emprendimiento social en la formación integral
}

FECHA DE RECEPCIÓN: 27 de julio

FECHA DE APROBACIÓN: 26 de agosto

pp. 175-190

DOI: http://dx.doi.org/10.21158/01208160.n81.2016.1554

\section{Resumen}

El presente artículo busca reconocer tendencias e intereses hacia la formulación de proyectos sociales desde la catedra ética para empresarios en la Unidad de estudio Constitución política, ética y responsabilidad social de la Universidad EAN, una temática que involucra diferentes tópicos como caracterización en la formación integral y el reconocimiento de oportunidades, que no se orientan únicamente por el afán de lucro, sino que operan dentro de sus propios contextos con criterios éticos definidos; esto significa el descubrimiento de la responsabilidad social que tiene toda organización.

La práctica educativa y social enfrenta nuevos retos para fortalecer la ética, la responsabilidad social, los valores universales en general, y para contribuir con los propósitos del desarrollo sostenible es necesario repensar los enfoques y las estrategias formativas e investigativas, a fin de vincularlas de manera práctica y útil a las realidades y demandas del entorno.

\section{Palabras clave}

Innovación social, Responsabilidad Social, ética para empresarios, educación social, ODS.

\section{David Ricardo Ocampo Eljaiek}

Magíster en Gestion Urbana, Universidad Piloto de Colombia; arquitecto Universidad, Piloto de Colombia. Docente asociado, Universidad EAN.

${ }^{1}$ La unidad de estudio Constitución Política, Ética y Responsabilidad Social hace parte de la oferta académica, del núcleo sociohumanísticas, adscrita a la Facultad de Humanidades y Ciencias Sociales de la Universidad EAN. 


\section{Social Entrepreneurship in Integral Training}

Abstrac. This article seeks to recognize trends and interests towards the formulation of social projects from the class named Ethics for entrepreneurs, topic Political Constitution, Ethics and Social Responsibility of the EAN University, a theme that involves different topics as a characterization in the integral formation and the recognition of opportunities, which are not only driven by the desire for profit, but operate within their own contexts with defined ethical criteria; this means the discovery of the social responsibility that every organization has.

Educational and social practice faces new challenges to strengthen ethics, social responsibility, universal values in general, and to contribute to the goals of sustainable development, it is necessary to rethink the approaches and the formative and investigative strategies, in order to link them in a practical way and make it useful to the realities and demands of the environment.

Key words. Social innovation, social responsibility, ethics for entrepreneurs, social education, ODS.

\section{Entrepreneuriat social et formation intégrale}

Resumé. Cet article tente de rendre compte des tendances et des intérêts de la formulation de projets sociaux et éthiques pour les entrepreneurs inscrits au cours de Constitution politique, Ethique et Responsabilité sociale de l'Université EAN. Cette thématique implique différents thèmes tels que la caractérisation de la formation intégrale et la reconnaissance d'opportunités ne s'orientant pas uniquement vers des perspectives purement lucratives mais qui agissent dans des contextes précis, grâce à des critères éthiques définis, ouvrant la voie à la prise en compte de la responsabilité sociale que possède toute organisation.

Les pratiques éducatives et sociales font face à de nouveaux défis pour rétablir l'éthique, la responsabilité sociale et plus généralement les valeurs universelles car, pour contribuer au développement durable, il devient nécessaire de repenser les objectifs et stratégies de formation et d'investigation pour les faire correspondre de manière pratique et utile aux réalités et aux attentes des sociétés.

Mots clefs. Innovation sociale, responsabilité sociale, éthique des entrepreneurs, éducation sociale, ODS.

\section{O empreendedorismo social na formação}

Resumo. Este artigo reconhece as tendências e interesses para a formulação de projetos sociais da aula ética para os empresarios, na unidade de estudo Constituição, ética e responsabilidade social da Universidade EAN, um tema que envolve temas diferentes, tais como a caracterização na formação e reconhecimento das oportunidades que não são voltadas apenas pelo lucro, mas operam dentro de seus próprios contextos com critérios éticos; isto significa a descoberta da responsabilidade social de qualquer organização.

A prática educativa e social enfrenta novos desafios para reforçar a ética, responsabilidade social, valores universais em geral, e para contribuir para os objectivos do desenvolvimento sustentável é necessário repensar abordagens e formação e estratégias de investigação, a fim de ligá-los de forma prática e útil às realidades e demandas do ambiente.

Palabras chave. Inovação social, responsabilidade social, ética para empresários, educação social, ODS. 


\section{Introducción}

Cuando se establecen retos éticos con compromisos sociales en el ámbito de lo público, lo privado y lo comunitario, el desarrollo personal y el desempeño profesional al conocimiento integral conducen a definir, según, Poveda, a «[...] analizar la realidad de la Responsabilidad Social Empresarial, desde un punto de vista especializado y sectorial, profundizando en las particularidades a las que se enfrentan los diferentes sectores» (2005), comprometiendo, desde su base, en la academia y la educación el implementar y comprender las dinámicas económicas, sociales y ambientales, con responsabilidad social y caracterizando y definiendo el destino de las sociedades contemporáneas.

Por esta razón, y en reacción al cuestionamiento ético, desde nuestra unidad de estudio, se considera necesario indagar en los procesos de aprendizaje los aspectos sociales e intereses frente a estos últimos, generando la reflexión en torno al papel y compromiso de los futuros profesionales en la formulación, puesta en operación de proyectos innovadores de carácter social y cómo estos podrían ser determinantes en la construcción de las organizaciones modernas, públicas y privadas, las cuales dan sentido a todo accionar en el marco del Estado colombiano.
En este sentido, y teniendo la oportunidad de contar con los estudiantes de los diferentes programas de la Universidad EAN - Institución académica fundada en el año de 1967 con el postulado de hacer del profesional un empresario-, en el espacio académico «Constitución Política Ética y Responsabilidad Social (CPERS)», unidad de estudio que aborda la complejidad del ser humano y la de sus potencialidades desde la perspectiva de la ética humanista, basada en los principios y valores e implícita en la misión de la Universidad en la que se menciona: «[...] Contribuir a la formación integral de la persona y estimular su aptitud emprendedora, de tal forma que su acción coadyuve al desarrollo económico y social de los pueblos» (Universidad EAN, 1969), se aborda el análisis de las comunidades políticas, en especial el colombiano, a partir del estudio de la Constitución Política de 1991, desde el punto de vista dogmático y haciendo un especial énfasis en los principios y valores que orientan el accionar de todos en el Estado Social de Derecho, así como las teorías éticas desde las cuales se promueve en la actualidad: el desarrollo económico, social y ambiental; la participación activa de los estudiantes; y la consolidación de la necesaria relación que existe entre el sector privado y el entorno social dentro de un cuadro de valores éticos universalmente reconocidos. 


\section{Emprendimiento social y la educación}

P

ara hacer un acercamiento al concepto, se da entrada a las reflexiones plateadas por Kliksberg (2013): la educación es la clave del siglo XXI, en un mundo con 1.100 millones de personas sin agua potable, 1.700 millones con hambre y un $20 \%$ de los jóvenes fuera del mercado del trabajo, «la única estrategia posible es la educación. Es así como, dentro de las teorías actuales sobre emprendimiento, la propuesta por Shane, que indica que «[...] Un emprendedor social podrá ser un empresario capaz de establecer soluciones a los problemas de una organización fortaleciendo las oportunidades del negocio (Shane, 2000), resalta una nueva conciencia en cuanto que las empresas no pueden ser islas, pues el enfoque Kliksberg toma la sociedad en su conjunto.

Las empresas requieren, pues, entender la problemática social que las rodea; para ello, tienen la oportunidad de servirse de la experticia de los emprendedores sociales, siendo este un desarrollo natural de cualquier economía, «[...] pero teniendo en cuenta los aspectos sociales e incluyentes de la sociedad así como identificar las competencias del personal brindando oportunidades a todos» (2000).

Si bien este trabajo se centra en el emprendimiento empresarial, en el mismo se puede identificar el desequilibrio que causa la exclusión, el sufrimiento humano, la creación del valor social, la innovación social, la proactividad, y los riesgos. Por su parte, en el trabajo de Shane, la definición de emprendimiento viene referida por la formulación puesta en operaciónevaluación de un negocio, la cual permite generar planteamientos donde la necesidad o la característica social estén presentes en cada idea de negocio. Partiendo de esta confluencia, se puede plantear la necesidad de construir empresa con un sentido social, y cómo orientar a la reflexión con base en las ya propuestas por Kliksberg: el futuro profesional responderá a las actividades de desarrollo de un territorio y sus dinámicas; adicionalmente, en el sentido amplio, la innovación social podría entender cómo hacer las cosas de manera diferente y novedosa, con acciones que ayuden a la sociedad a establecer y cumplir objetivos tomando riesgos con un carácter proactivo (Figura 1). 
Figura 1. Emprendimiento social

\begin{tabular}{|c|c|c|}
\hline \multirow{3}{*}{$\begin{array}{l}\text { Universidad EAN } \\
\text { Formación integral }\end{array}$} & Creación de valor compartido & \multirow[b]{2}{*}{ Proactividad } \\
\hline & \multirow{2}{*}{$\begin{array}{l}\text { Se entiende como creación de renta o ri- } \\
\text { queza. También puede ser entendido como } \\
\text { la careación de algo nuevo con valor. }\end{array}$} & \\
\hline & & \multirow{3}{*}{$\begin{array}{l}\text { Se entiende asíla actitud que toma } \\
\text { el pleno control de la conducta } \\
\text { de una manera activa, tomando } \\
\text { la iniciativa en el desarrollo de } \\
\text { acciones y la responsabilidad de } \\
\text { dichas acciones. }\end{array}$} \\
\hline & Circunstancia empresarial & \\
\hline \multirow[t]{2}{*}{$\begin{array}{l}\text { Constitución política ética } \\
\text { y responsabilidad social }\end{array}$} & $\begin{array}{l}\text { Viene definido como el momento propicio } \\
\text { para hacer algo, tanto a nivel temporal, a } \\
\text { nivel locativo y a nivel coyuntural. }\end{array}$ & \\
\hline & Innovación social & Riesgos \\
\hline Emprendimiento social & $\begin{array}{l}\text { En sentido amplio se puede entender cómo } \\
\text { hacer las cosas de manera diferente y } \\
\text { novedosa, en sentido estricto sería la intro- } \\
\text { ducción de un nuevo producto, servicio o } \\
\text { procedimiento. }\end{array}$ & $\begin{array}{l}\text { Se refiere a la toma de acciones } \\
\text { con resultados inciertos. En mu- } \\
\text { chos casos está también asociada } \\
\text { a la toma de decisiones en situa- } \\
\text { ciones complejas. }\end{array}$ \\
\hline
\end{tabular}

Fuente. Elaboración propia del autor.

La anterior información permite reconocer los elementos del emprendimiento social y cómo este se articula en la unidad de estudio CPERS identificando el valor compartido, las circunstancias empresariales y la innovación social, además de los riesgos que están en manos de los emprendedores sociales. Esto lo pone en consideración Grau en el segundo capítulo de su cuaderno "Organización y gobernanza de las empresas sociales», en el que indica que: "[...] Cabe precisar que no todas las empresas sociales llevan a cabo actividad emprendedora, ya que esto depende del grado de innovación del proyecto» (2012), de sus ilusiones y de su educación, especialmente la superior, afrontando los importantes retos de emprender innovando y de abrir nuevos caminos, única manera de lograr el éxito: transformar la sociedad y equilibrar el desarrollo de nuestras sociedades.

Adicionalmente, según la Ley 1014 de 2006, artículo $1^{\circ}$ del Congreso de la República de Colombia (2006), el emprendimiento es una forma de pensar, razonar y actuar centrada en las oportunidades, planteada con visión global y llevada a cabo mediante un liderazgo equilibrado y bajo la gestión de un riesgo calculado; su resultado es la creación de valor que beneficia a la empresa, la economía y la sociedad.

Mort, indica en su artículo Investigating social entrepreneurship, que el emprendimiento, que surgió hace poco tiempo, es: a multidimensional model; además, afirma que «el término emprendedor se originó en la economía francesa entre los siglos XVII y XVIII, y sugiere que el emprendedor desplaza fondos de un área de mayor disponibilidad para llevarlos a un área de mayor productividad y grandes rendimientos» (2005).

El emprendimiento, con frecuencia, es definido como la búsqueda oportunista de riqueza a través de iniciativas creativas de operación individual, en un entorno restringido por la limitación de recursos (Austin et al., 2006; Mitchell et al., 2002; Nga y Shamugathan, 2010), considerando que Kliksberg integra elementos con causas y consecuencias.

En tal sentido, Kliksberg, en el libro «Emprendedores sociales», reconoce la necesidad indebida que causa el rechazo, la pobreza y desventura de la particularidad del ser 
humano el cual no tiene la posibilidad de lograr, por sí solo, transformaciones de su realidad situacional. Por ello, invita al emprendedor social a buscar la oportunidad de cambiar esa difícil situación, creando un valor social con innovación que genere equilibrio y que le asegure una mejor condición que contribuya a la sociedad.

\section{Las competencias de los emprendedores sociales}

liksberg, construye seis grupos de competencias: axiológicas, modos de pensar, actitudes, capacidades de convocatoria, orientación a la acción y construcción de alianzas estratégicas, y redes (Figura 2).

Figura 2. Unidades temáticas

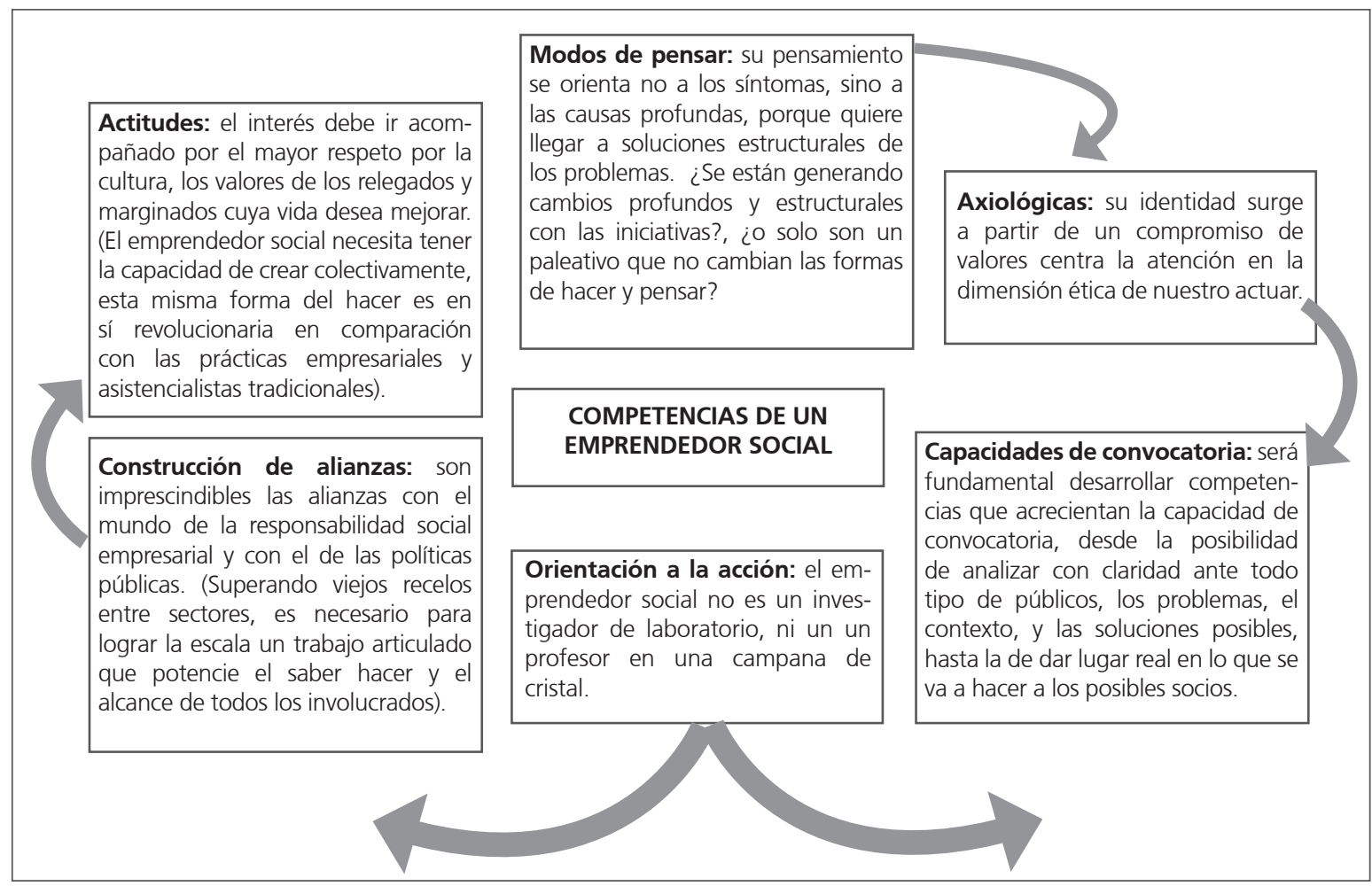

Fuente. Kligsberg, (s.f).

De esta manera, Kliksberg, propone las siguientes 16 unidades temáticas que hacen la formación de los emprendedores sociales. 


\section{Marco metodológico}

U

na vez analizados el alcance y los objetivos

trazados, se enmarcó dentro de un estudio tomando como partida la unidad de estudio de referencia CPERS (Hernández, 2003). Para su realización, se aplicó el instrumento tipo encuesta comprendido en las siguientes fases:

\section{- Fase I: recopilación y verificación de información}

- Documentación general: se realizó un análisis general de la unidad de estudio con el propósito de lograr un acercamiento al estado de la oferta y los intereses en la población frente al tema; búsqueda de información con

base en la unidad de estudio «Constitución política, ética y Responsabilidad Social», y sus temáticas.

\section{- Fase II: aplicación encuesta}

- Público objeto del estudio: se aplicó la encuesta directa por medio de un cuestionario semiestructurado (Sampieri et al., 2003), dirigido a estudiantes que cursan la unidad de estudio «Constitución política, ética y Responsabilidad Social», de forma aleatoria y distribuida por horarios según los siguientes grupos: tres, seis, siete, doce y trece, de un total de 14 grupos ofrecidos en el ciclo; estos están resaltados en color amarillo (Tabla 1).

Tabla 1. Oferta unidad de estudio 2016 c2

\begin{tabular}{|c|c|c|c|c|c|c|c|c|c|c|c|c|c|c|c|}
\hline $\begin{array}{l}\text { CódIGo } \\
\text { UNIDAD DE } \\
\text { ESTUDIO }\end{array}$ & NOMBRE UNIDAD DE ESTUDIOS & $\begin{array}{l}\text { NÚCLEO DE } \\
\text { FORM. }\end{array}$ & $\begin{array}{c}\text { PLANES DE } \\
\text { ESTUDIO } \\
\text { PARALOS } \\
\text { CUALES SE } \\
\text { OFERTA } \\
\end{array}$ & $\begin{array}{l}\text { NÚMERO DE } \\
\text { CRÉDTOS }\end{array}$ & $\begin{array}{c}\text { HORAS } \\
\text { SEMESTRALES POR } \\
\text { UNIDADES DE } \\
\text { ESTUDIO }\end{array}$ & GRUPO & cupo & \begin{tabular}{|c|} 
NÚMERO DE \\
ESTUDIANT \\
ES \\
MATRICULA \\
DOS \\
\end{tabular} & $\begin{array}{c}\text { CICLOII } \\
\text { SEMESTR } \\
\mathrm{E} \\
\end{array}$ & L & M & Mc. & 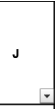 & $\mathbf{v}$ & . \\
\hline 002234 & $\begin{array}{l}\text { CONSTTUCIÓN POLIIICA ÉTICAY } \\
\text { RESPONSABLIDAD SOCCIAL }\end{array}$ & $\begin{array}{l}\text { SOCIO } \\
\text { HUMANSTICO }\end{array}$ & 144 & 3 & 54 & 1 & 30 & 31 & C2 & 7:00 A 9:00 & & 7:00 A 9.00 & & 7:00 A9:00 & \\
\hline 002234 & $\begin{array}{l}\text { CONSTTUCIÓN POLIITICA ÉTICAY } \\
\text { RESPONSABLIDAD SOCCIAL }\end{array}$ & $\begin{array}{l}\text { SOCIO } \\
\text { HUMANISTICO }\end{array}$ & 144 & 3 & 54 & 2 & sо & 32 & c2 & 7:00 A9:00 & & 7:00 A 9:00 & & 7:00 A9:00 & \\
\hline 002234 & $\begin{array}{l}\text { CONSTTUCION POLITICA ÉTICAY } \\
\text { RESPONSABLIDAD SOCGLL }\end{array}$ & $\begin{array}{l}\text { SOCIO } \\
\text { HUMANISTICO }\end{array}$ & 144 & 3 & 54 & 3 & 30 & э3 & c2 & $9: 00$ A $11: 00$ & & $9: 00$ A $11: 00$ & & 9:00 A $11: 00$ & \\
\hline 002234 & $\begin{array}{l}\text { CONSTTUCION POLITICA ĖTICAY } \\
\text { RESPONSABLIDAD SOCGL }\end{array}$ & $\begin{array}{l}\text { SociO } \\
\text { HUMANISTICO }\end{array}$ & 144 & 3 & 54 & 4 & 30 & 29 & c2 & $9: 00$ A $11: 00$ & & 9:00 A 11:00 & & 9:00 A 11:00 & \\
\hline 002234 & $\begin{array}{l}\text { CONSTTUCION POLITICA ÉTICAY } \\
\text { RESPONSABLIDAD SOCCAL }\end{array}$ & $\begin{array}{c}\text { SOCIO } \\
\text { HUMANISTICO }\end{array}$ & 144 & 3 & 54 & 5 & 30 & 34 & c2 & 11:00 A $1: 00$ & & 11:00 A 1:00 & & 11:00 A 1:00 & \\
\hline 002234 & $\begin{array}{l}\text { CONSTITUCIÓN POLITICA ÉTICAY } \\
\text { RESPONSABIIIDAD SOCGIAL }\end{array}$ & $\begin{array}{c}\text { SOCIO } \\
\text { HUMANISTICO }\end{array}$ & 144 & 3 & 54 & 6 & 30 & 23 & c2 & 2:00 A 4:00 & & $2: 00$ A 4:00 & & 2:00 A 4:00 & \\
\hline 002234 & $\begin{array}{l}\text { CONSTITUCIÓN POLIIICA ÉtIICAY } \\
\text { RESPONSABLIDAD SOCGAL }\end{array}$ & $\begin{array}{l}\text { SOCIO } \\
\text { HUMANISTICO }\end{array}$ & 144 & 3 & 54 & 7 & 30 & 25 & $\mathrm{C} 2$ & 6:00 A8:00 & & 6:00 A 8:00 & & 6:00 A 8:00 & \\
\hline 002234 & $\begin{array}{l}\text { CONSTTUCIÓN POLIITCA ÉTICAY } \\
\text { RESPONSABLIDAD SOCIAL }\end{array}$ & $\begin{array}{l}\text { SOCIO } \\
\text { HUMANISTICO }\end{array}$ & 144 & 3 & 54 & 8 & 30 & 34 & C2 & $8.00 \mathrm{~A} 10.00$ & & $8.00 \mathrm{~A} 10.00$ & & $8.00 \mathrm{~A} 10.00$ & \\
\hline 002234 & $\begin{array}{l}\text { CONSTTUCIÓN POLIITCA ÉTICAY } \\
\text { RESPONSABLIDAD SOCGAL }\end{array}$ & $\begin{array}{l}\text { SOCIO } \\
\text { HUMANISTICO }\end{array}$ & 144 & 3 & 54 & 9 & 30 & 24 & c2 & 7:00 A 9:00 & & $7: 00$ A $9: 00$ & & 7:00 A9:00 & \\
\hline 002234 & $\begin{array}{l}\text { CONSTTUCIÓN POLIITLA ÉTICAY } \\
\text { RESPONSABLIDAD SOCGLL }\end{array}$ & $\begin{array}{c}\text { SOCIO } \\
\text { HUMANISTICO }\end{array}$ & 144 & 3 & 54 & 10 & so & sо & c2 & 7:00 A 9:00 & & 7:00 A 9:00 & & 7:00A9:00 & \\
\hline 002234 & $\begin{array}{l}\text { CONSTTUCIÓN POLITICA ÉTICAY } \\
\text { RESPONSABLIDAD SOCGLL }\end{array}$ & $\begin{array}{c}\text { SOCIO } \\
\text { HUMANISTICO }\end{array}$ & 144 & 3 & 54 & 11 & 30 & 22 & c2 & $9: 00$ A $11: 00$ & & $9: 00 \mathrm{~A} 11: 00$ & & 9:00 A 11:00 & \\
\hline 002234 & $\begin{array}{l}\text { CONSTTUCION POLITICA ÉTICAY } \\
\text { RESPONSABLIDAD SOCGL }\end{array}$ & $\begin{array}{l}\text { SOCIO } \\
\text { HUMANISTICO }\end{array}$ & 144 & 3 & 54 & 12 & 30 & 32 & c2 & 9:00 A 11:00 & & $9: 00 \mathrm{~A} 11: 00$ & & 9:00 A 11:00 & \\
\hline 002234 & $\begin{array}{l}\text { CONSTIUUCION POLIITCA ÉTICAY } \\
\text { RESPONSABIIDAD SOCIAL }\end{array}$ & $\begin{array}{l}\text { SOCIO } \\
\text { HUMANISTICO }\end{array}$ & 144 & 3 & 54 & 13 & 30 & 34 & C2 & 11.00 A 1.00 & & 11:00 A $1: 00$ & & $11: 00 \mathrm{~A}: 00$ & \\
\hline 002234 & $\begin{array}{l}\text { CONSTIUUCIÓN POLIIICA ÉtIICAY } \\
\text { RESPONSABIIDAD SOCIALL }\end{array}$ & $\begin{array}{c}\text { SOCIO } \\
\text { HUMANISTICO }\end{array}$ & 144 & 3 & 54 & 14 & 30 & 17 & $\mathrm{C} 2$ & Z:00 A 4:00 & & 2:00 A 4:00 & & 2:00 A 4:00 & \\
\hline
\end{tabular}

Fuente. Programación académica sociohumanísticas V3. 2016 s1-c3 
Se construyó la siguiente ficha técnica (Tabla 2); para calcular el tamaño de la muestra se utilizó la fórmula de Balestrini (1999) para poblaciones finitas, aplicada a 40 hombres y 55 mujeres entre los 15 y 40 años; estudiantes que cursan la unidad de estudio CPERS, es decir, un promedio de 450 estudiantes por semestre; por lo tanto, el tamaño de la muestra, como mínimo a encuestar, fue de 95 estudiantes.

Tabla 2. Ficha técnica

\begin{tabular}{|l|l|}
\hline Diseño muestra. & Fórmula de Balestrini (1999) para poblaciones finitas. \\
\hline Población objetivo. & $\begin{array}{l}40 \text { hombres y } 55 \text { mujeres entre los } 15 \text { y } 40 \text { años, } \\
\text { estudiantes que cursan la unidad de estudio CPERS. }\end{array}$ \\
\hline Universo representado. & $\begin{array}{l}420 \text { estudiantes promedio por semestre que toman } \\
\text { la Unidad de estudio. }\end{array}$ \\
\hline Tipo de estudio y tipo de investigación. & Estadístico/cuantitativo. \\
\hline Técnica & Aplicación de encuesta en clase diferentes grupos. \\
\hline Tipo de muestreo. & Muestreo aleatorio simple. \\
\hline Tamaño de muestra. & 95 estudiantes. \\
\hline Momento estadístico. & Abril y mayo de 2016. \\
\hline Margen de error observado. & $2 \%$ \\
\hline Nivel de confianza. & $98 \%$ \\
\hline Persona que la realizó. & David Ricardo Ocampo Eljaiek. \\
\hline Tema al que se refiere. & Percepción catedra ética para empresarios CPERS. \\
\hline preguntas que se formularon. & (Diez preguntas) ver anexo. \\
\hline
\end{tabular}

Fuente. Elaboración propia del autor a partir de Balestrini (s.f).

De los encuestados, 64 estudiantes corresponden a Lenguas Modernas, seguido por Ingeniería Química, Negocios Internacionales, Administración de Empresas, Economía e Ingeniería Ambiental, de los programas de pregrado presencial (Figura 3).

La población se distribuye aleatoriamente en los estudiantes emprendedores e intraemprendedores, como lo menciona Medina (2011), predominando las mujeres, en su gran mayoría del programa de Lenguas Modernas; el 47\%, corresponde a estudiantes de primer semestre, segundo, cuarto, quinto, sexto y octavo, respectivamente. Se anota que la unidad de estudio corresponde a créditos que son tomados durante el desarrollo del programa, por eso los diferentes semestres en la unidad de estudio; esto corresponde a la posibilidad y la flexibilidad que permite ver dicha unidad durante la carrera, pero en especial orden de los primeros semestres, pues es el momento de formar integralmente y bajo los principios de valores de la Universidad (Crissien, 2010). 
Figura 3. Estudiantes representados en los programas que ofrece la Universidad EAN.

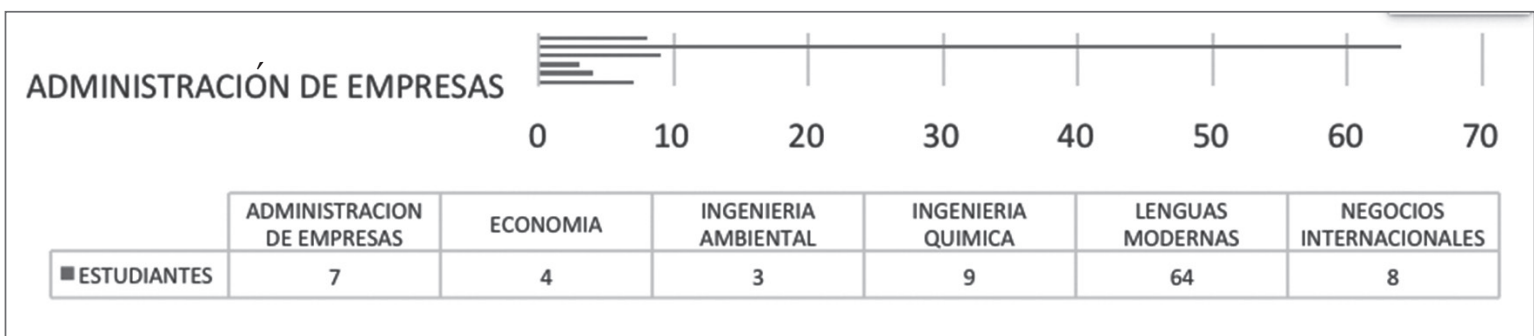

Fuente. Elaboración propia del autor.

\section{Procesamiento y análisis de la información —}

Dosteriormente, cada una de las encuestas fue digitalizada y definida desde el plan de muestreo; las necesidades fueron estipuladas en el plan de análisis e ingresadas a un software estadístico Excel que arroja la información depurada y la traslada a tablas y gráficas.

La información obtenida de la realización de las encuestas a una muestra significativa de la unidad de estudio fue tabulada y representada en gráficas de tal forma que permitió realizar

Figura 4. Formato de encuesta aplicado

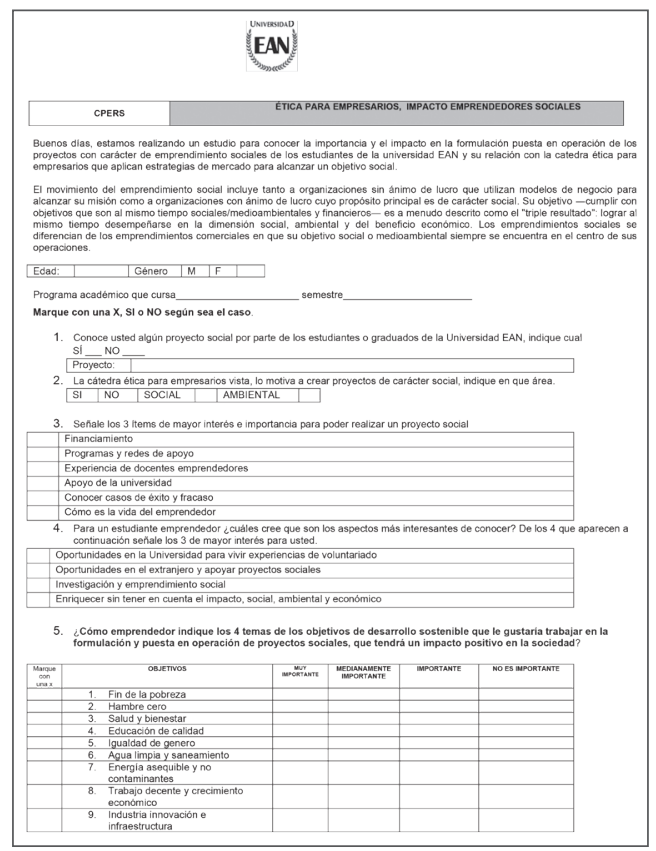

Fuente. Elaboración propia del autor. un análisis de los resultados. Se procederá, así, a determinar los pasos a seguir en la investigación (Ocampo, 2015).

\subsection{Cuerpo de la encuesta}

Se elaboró una encuesta para realizar en el espacio académico, con diez preguntas representadas en un formato, el cual reposa en los anexos base de la investigación (Figura 4).

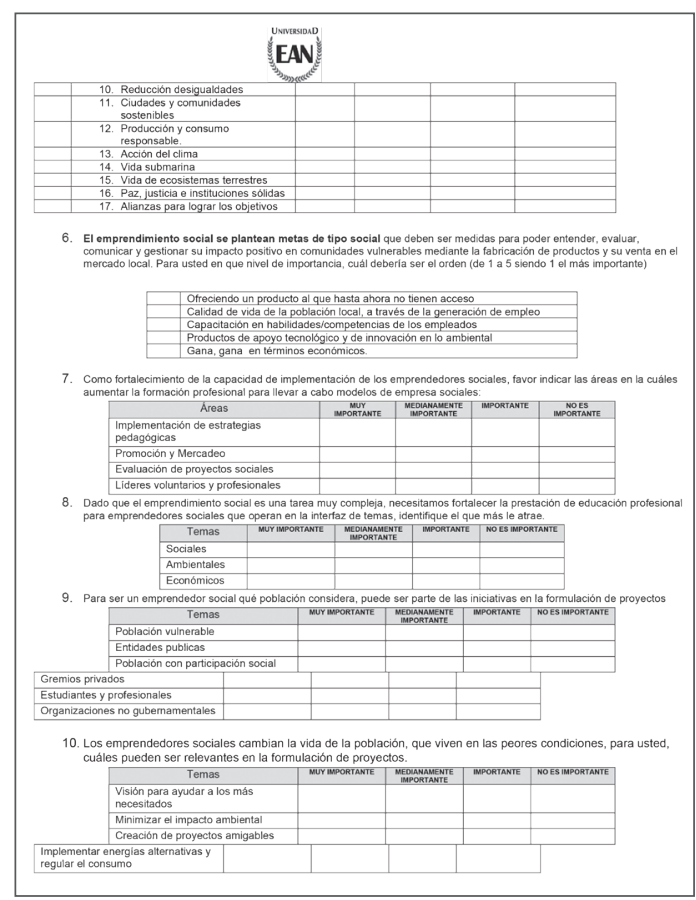




\section{Resultados y discusión}

P artiendo de la encuesta realizada, la cual fue el instrumento de medición, se recopiló una serie de datos para el desarrollo del trabajo de investigación, información de interés para determinar factores claves (Figura 5).

Figura 5. Resultados a la pregunta: ¿Qué proyectos sociales creados desde la Universidad conoce?

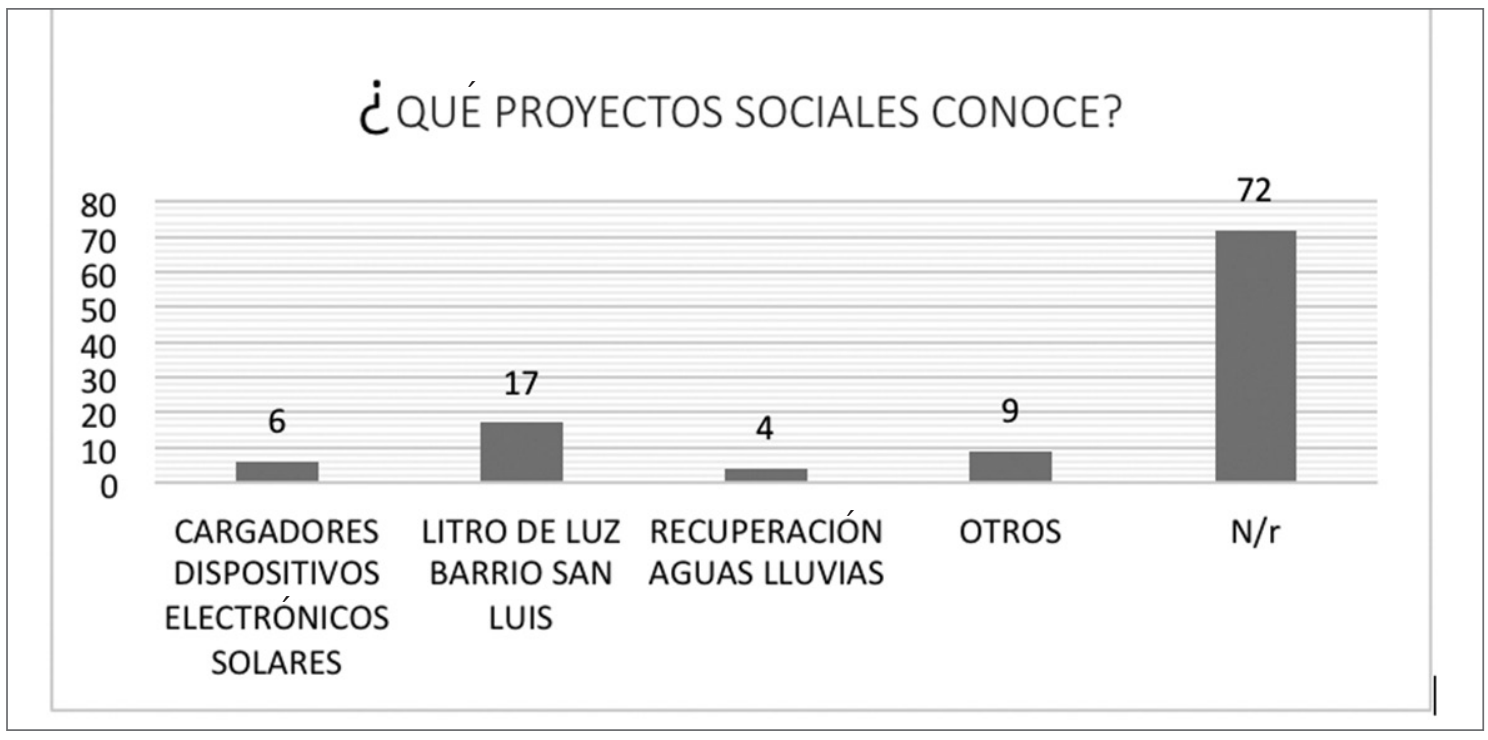

Fuente. Elaboración propia del autor.

El proyecto que más reconocimiento tuvo fue el siguiente:

- Diecisiete estudiantes conocieron el proyecto de iluminación «Litro de luz»² en el barrio San Luis, Municipio La Calera ${ }^{3}$, Cundinamarca; en este, toda la comunidad académica usó los cargadores solares instalados en la Plazoleta Emprendedores, de la Universidad EAN, así como el agua lluvia en los sanitarios de esta misma Institución. Para ello, la Universidad realizó, en diferentes unidades de estudio, la visita al Canopy urbano, un laboratorio sostenible ubicado en el octavo piso del edificio de la Sede El Nogal, permitiendo identificar los proyectos y apropiarlos como referentes instituciones.

${ }^{1}$ La fundación Un Litro de Luz Colombia nace en el año 2011 como réplica oficial del proyecto Liter of Light que se llevaba a cabo desde algunos meses atrás en Filipinas. Un modelo de intervención social y trabajo comunitario eran el soporte de las instalaciones de "Las Botellas de Moser", que no son más que botellas de 1,5 litros llenas de agua y con 10 mililitros de Cloro, que al instalarse en los techos permite refractar la luz solar dentro de un espacio que carece de iluminación.

2 El Municipio La Calera es un poblado indígena que se denominaba Teusacá. Según algunos historiadores, significa "prisión", y según Acosta Ortegón es "cercado prestado". Por el año de 1597, los indígenas no estaban radicados en Teusacá ni tenían iglesia, de acuerdo con la visita que hizo el Oidor Ibarra. Por Auto de fecha $1^{\circ}$ de septiembre de 1604, el Oidor Lorencio de Terrones ordenó juntar los indios de Teusacá, con un total de 359 distribuidos en las parcialidades de Teusacá, Suabso, Tabtiba, Suto y Tuchasgula. 
Ante la pregunta: ¿la unidad de estudio Constitución política, ética y Responsabilidad Social (CPERS), lo motiva a crear proyectos de carácter social? Se evidencia un alto margen de interés, particularmente en el aspecto ambiental con 56 estudiantes atraídos por estos temas; le sigue el interés en los proyectos de carácter social con 39 estudiantes, confirmando la competencia general de la unidad de estudio donde el estudiante identifica, analiza y establece una posición crítica en torno al obrar ético de los seres humanos en los ámbitos de lo público y lo privado, en especial, a la práctica de la Res-ponsabilidad Social con relación a los valores, principios y normas constitucionales bajo un interés social, ambiental y económico (Figura 6).

Figura 6. Resultado de la pregunta ¿la unidad de estudio Constitución Política, Ética y Responsabilidad Social lo invita a crear proyectos de carácter social?

\begin{tabular}{|c|c|}
\hline \multirow{m}{*}{} & $\stackrel{\circ}{\text { in }}$ \\
\hline SOCIAL & AMBIENTAL \\
\hline
\end{tabular}

Fuente. Elaboración propia autor.

En el siguiente enunciado se le indica al encuestado: señale tres ítems de mayor interés e importancia para poder realizar un proyecto social (Figura 7).

Figura 7. Resultados enunciado: señale tres ítems de mayor interés e importancia que inviten a realizar un proyecto social

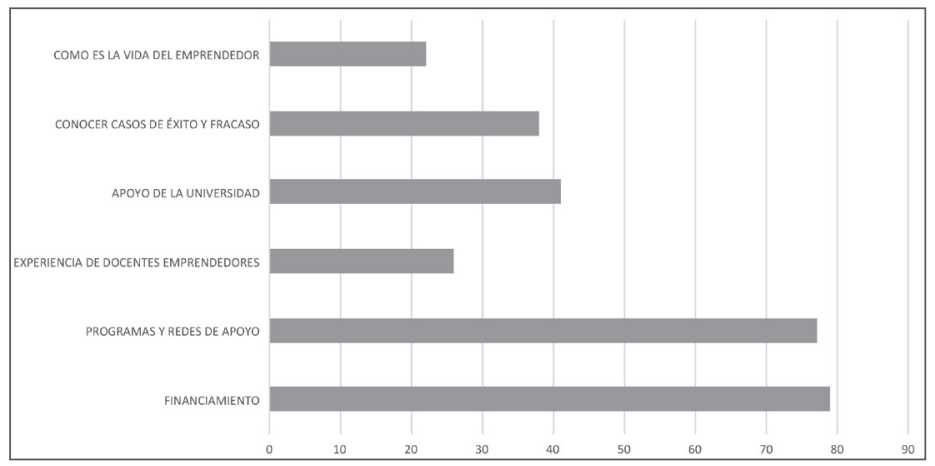

Fuente. Elaboración propia del autor.

Los encuestados marcaron como primer ítem, el financiamiento, evidenciando el interés generalizado en la formulación de proyectos sociales y ambientales lo que coincide con el texto del Banco mundial: «[.. ] El Banco Mundial se redimensiona con el objetivo de estar cónsono con un desarrollo sustentable de los países a los que financia» (Kim, 2016). Se destaca que los encuestados ven en la financiación un ítem importante con 79 participaciones directas, así como la identificación de programas con los actores y redes involucrados que brinden apoyo. Así mismo, ven en la Universidad un actor importante en cuanto al apoyo, pues les da la posibilidad de usar los espacios académicos y físicos en la formulación de los mismos. Para los emprendedores es importante tener presente los casos de éxito o fracaso, pues esto termina siendo una necesidad de aprendizaje así como la experiencia del docente (Figura 8). 
Figura 8. ¿Cuáles cree que son los aspectos más interesantes de conocer?

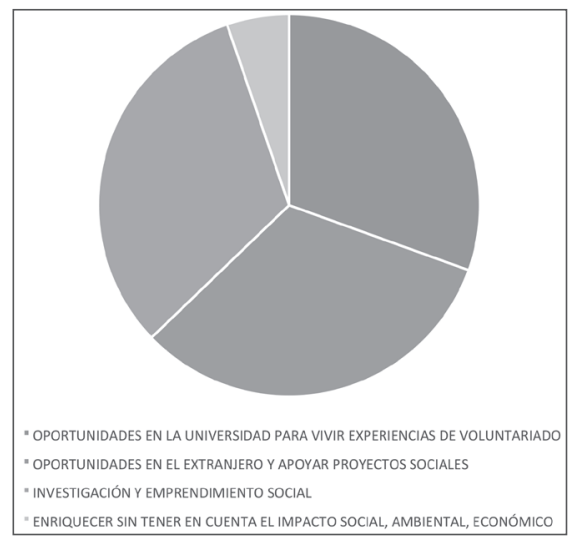

Fuente. Elaboración propia del autor.

Llama la atención que, con un promedio generalizado en la pregunta anterior, el $32 \%$ considera importante tener experiencias en el extranjero y apoyar proyectos sociales, en especial en territorios con problemas en este aspecto; esto podría estar asociado a la carrera de Lenguas Modernas. De igual forma, con un empate del $32 \%$, los encuestados consideran que pueden tener ingresos, sin embargo, contemplan el impacto social y ambiental; además, el $31 \%$ considera que tiene el deseo de vivir una experiencia de voluntariado desde la práctica, siendo este un interés potencial en los estudiantes.

Como dato de interés, se presenta la siguiente pregunta: teniendo en cuenta que los emprendedores sociales cambian la vida de la población ¿qué vive en las peores condiciones: para usted? ¿Cuáles pueden ser relevantes en la formulación de proyectos teniendo en cuenta los objetivos de desarrollo del milenio de la ONU (2014)? (Figura 9).

Figura 9. ¿Cuáles pueden ser relevantes en la formulación de proyectos teniendo en cuenta Ios ODS?

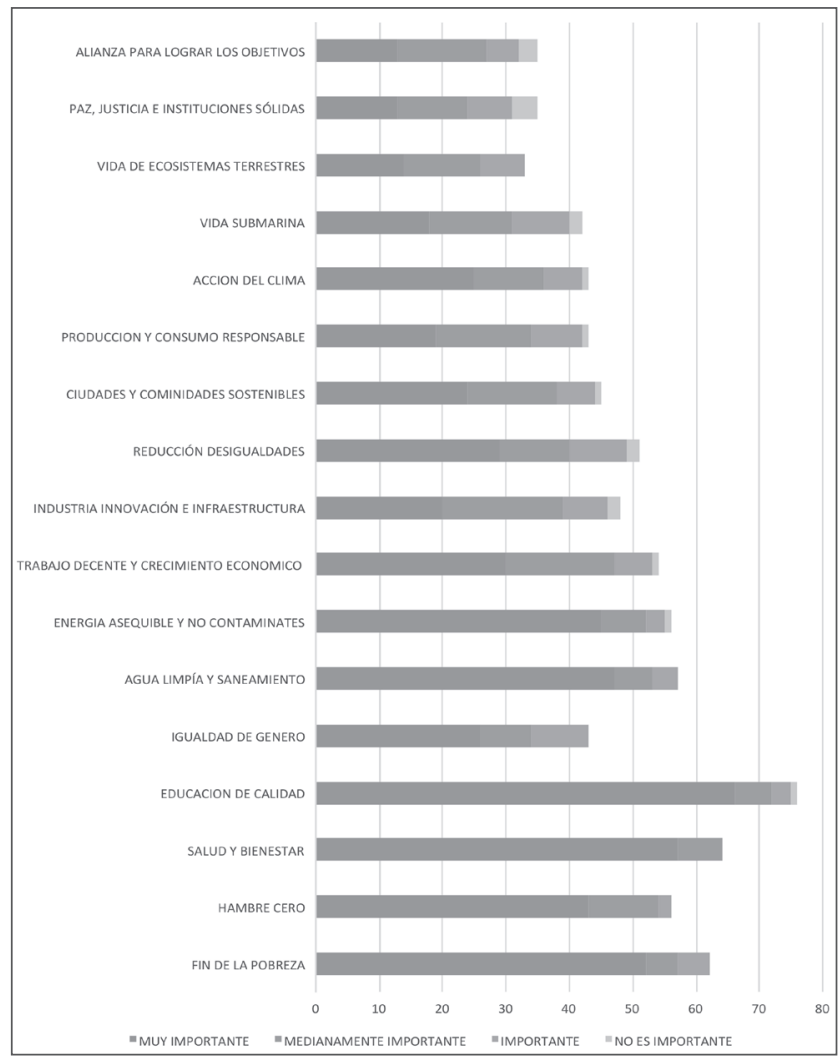

Fuente. Elaboración propia del autor. 
Con una respuesta favorable, 66 estudiantes consideran muy importante trabajar en los temas y desafíos universales que requieren acción global, así como lo referente a la educación de calidad inclusiva que se basa en la firme convicción de que la educación es uno de los motores más poderosos para garantizar el desarrollo sostenible teniendo como base la importancia de la formación; le siguen 57 estudiantes que consideran importante la salud y bienestar con cobertura universal, lo que incluye facilitar medicamentos y vacunas seguras y eficaces para todos: una parte esencial de este proceso es apoyar la investigación y el desarrollo de vacunas, además de proporcionar acceso a medicamentos. Lo anterior muestra el interés de estudiar siempre y cuando se tengan las condiciones ideales. El fin de la pobreza es el siguiente tema destacado, con una participación de 52 encuestados que consideran que este factor es un elemento importante para trabajar o formular proyectos: lo demuestran 43 opiniones que respaldan el ítem de hambre cero; agua y saneamiento están articulados con 47 puntos; y le sigue el interés de manejar energías asequibles y no contaminantes (Figura 10).

Figura 10. El emprendimiento social se plantea metas de tipo social

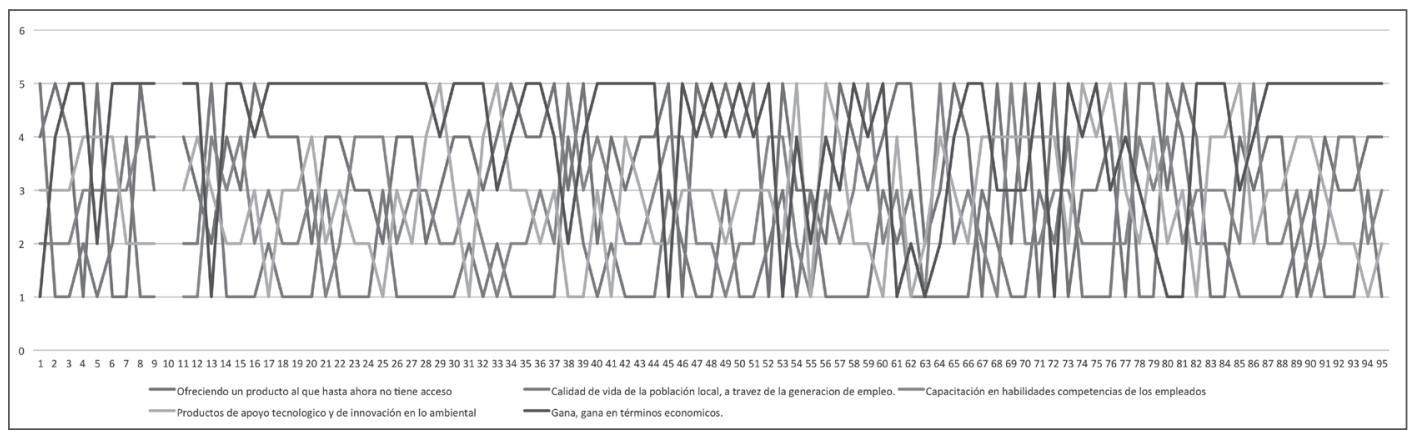

Fuente. Elaboración propia del autor.

El resultado sorprende pues pareciera que existe un interés entre el gana gana, en términos económicos, ya que manifiesta la formación y el interés de formular proyectos en los que el equilibrios o impacto sea compartido.

Como siguiente característica está la calidad de vida a través de la generación de empleo, articulando los productos de apoyo tecnológico y de innovación, en lo ambiental. Existen elementos correlacionales durante la aplicación, quedando en los últimos lugares de importancia ofrecer un producto al que, hasta el momento, no se tiene acceso, pues se asume que en esta época se tienen todos los elementos. En último nivel esta la capacitación, pues se asume que esta vendrá de forma autónoma.

Como es una aplicación inicial, para esta fase se determinó el interés, con las limitantes de los tiempos de aplicación. Se recomienda realizar la encuesta a los egresados para poder medir el impacto. A pesar del interés del emprendimiento social, se hace necesario, para una segunda etapa, incluir con más detalle los avances de los objetivos de desarrollo sostenible. 


\section{Conclusiones}

$\mathrm{T}$ ras realizar este estudio se puede concluir que los temas de emprendimiento social (Melián, 2011) cuentan con un perfil altamente diferenciado y articulado con los objetivos de desarrollo sostenible, lo que provoca que la mayoría de los estudiantes se involucren en actividades sociales a través de políticas de Responsabilidad Social. Es así como se evidencia que no se trata de luchar contra la pobreza, sino de trabajar en la productividad como una solución.

Se identificó que el emprendimiento social cuenta con un esquema de discusión y reflexión durante cuatro ciclos académicos equivalentes a un año y a tres créditos del plan de estudio; estos inician desde el mes de enero y finalizan en diciembre. Durante este proceso de formación se abarcan diferentes temáticas que incluyen el desarrollo de competencias investigativas, tecnológicas, normativas y sociohumanísticas que se construyen con la temática; en este sentido, se puede decir que dichas temáticas permiten a la Institución mantener unos estándares de calidad y cumplir con la formación integral de los futuros empresarios e intraempresarios que se forman en la Universidad EAN.
El conocimiento es necesario para incursionar en el emprendimiento social, así como el «[...] emprendimiento, ha cobrado interés para las escuelas de negocios desde la década de los ochenta» (Guzmán Vásquez, 2008), puesto que el $100 \%$ de los encuestados consideran necesario formular proyectos que generen impacto positivo en la sociedad gracias a la implicación de emprendedores que han visto en él la oportunidad de crear un negocio sostenible, es decir, capaz de generar los recursos necesarios para satisfacer sus costes, y a la vez, reinvertirlos para cubrir las necesidades de la sociedad generando el menor impacto ambiental posible.

Con relación a los atributos del emprendimiento social (Rojas, 2013), los proyectos realizados por iniciativa institucional hacen parte del referente colectivo; el emprendimiento social surge como iniciativa en la formación integral, pretendiendo dar respuesta a las necesidades reales de bienestar social y a las dificultades concretas de distintos colectivos.

El estudio refleja, la necesidad de consolidar los temas y desafíos universales que requieren acción global. 


\section{Referencias bibliograficas}

Congreso. (1991). Constitución poltíca de la República de Colombia. (C. d. República, Ed.) Bogotá, Colombia: Gaceta Constitucional, Vol. 1.

Crissien, C. (2010). Principiosyvalores Universidad EAN.Bogotá, Colombia: Universidad EAN.

Grau C, M.(2012). Cuadernos de la Cátedra "la Caixa" de Responsabilidad Social de la Empresa Gobierno Corporativo (Vol. 14). Navarra, España: Business School.

Guzmán Vásquez, A. (2008). Emprendimiento social - revisión de literatura. Estudios gerenciales, 24(109), pp. 1- 21.

Hernández S., (2003). Enfoque Cualitativo y uantitativo Metodología de la investigación. México: Editorial McGraw-Hill.

Kim, J. Y. (2016). Banco Mundial se redimensiona con nuevo marco social y ambiental. El Emprendedor, p. 1.

Kliksberg, B. (2012). Página 12. Recuperado de: www.pagina12.com.ar: www.pagina12. com.ar

Kliksberg, B. (2013). XV Congreso Mundial de Educación Comparada. Buenos Aires: Télam, p. 25. Recuperado de: http://www.telam. com.ar/notas/201306/22458-kliksberg-laeducacion-es-la-gran-lucha-que-tiene-quedar-el-mundo.php

Kliksberg, B. (2016). www. bernardokliksberg. com. Recuperado de: www. bernardokliksberg.com

Ministerio de Industria y Comercio (2006). Ley 1014 del Fomento de la Cultura del Emprendimiento. (s.c) (s.e).

Ocampo, D. (2015). Migración en la ciudad impacto urbano, escenarios de sostenibilidad. Revista EAN, 78(78), pp. 140-159.
Medina J. (2011). El intra-emprendedor: la innovación desde adentro. (s.c): América Económica.

Melián, A. (2011). Emprendimiento social y empresas de inserción. REVESCO, 106. Recuperado de: http://search.proquest.com/ openview/735a822a76e310878d201866a3 $8823 \mathrm{~cd} / 1$ ?pq-origsite=gscholar

Ministerio de Industria y Comercio (2006). Ley 1014 del Fomento de la Cultura del Emprendimiento. (s.c) (s.e).

ONU. (2014). Transformar nuestro mundo: la Agenda 2030 para el Desarrollo Sostenible. New York: ONU.

Poveda, R. G. (2005). Emprendimientos sociales en sectores estrategico. Bogotá D.C., Colombia: Gente Nueva Editores Ltda., Vol. 1, doi:978-958-98170-9-4

Rojas, G. R. (2013). Gerencia de la responsabilidad social en las organizaciones de hoy. Recuperado de: http://bdonline.ean. edu.co:2055/lib/bibliotecaeansp/reader. action?doclD=10933829:

Shane, S. (2000). Thepromiseofentrepreneurship asafieldofresearch. Academyofmanagement review. UniversitY of Mclrylcrnd ed., 25(1), 217-226., Virginia, USA: Academy ol Managemenl fleview. Recuperado de: http:// entrepreneurscommunicate.pbworks.com/f/ Shane\%2520\%252B\%2520Venkat\%2520\%2520Ent\%2520as\%2520field.pdf

Sullivan Mort, G. (2005). Investigating social entrepreneurship:A multidimensional model. Journal of World Business, pp. 21- 35.

Universidad EAN. (1969). Misión y visión de la Universidad EAN. Recuperado de: http:// institucion.universidadean.edu.co/seccion/ mision-y-vision-universidad-ean.html 